\title{
Design of a Coplanar Waveguide-to-Ridge Gap Waveguide Transition Via Capacitive Coupling
}

\author{
Astrid Algaba Brazález, Ashraf Uz Zaman, Per-Simon Kildal \\ Department of Signals and Systems \\ Chalmers University of Technology \\ Gothenburg, Sweden \\ astrid.algaba@chalmers.se, zaman@chalmers.se,per-simon.kildal@chalmers.se
}

\begin{abstract}
A transition from Coplanar Waveguide (CPW) via capacitive coupling in order to provide feeding to the so-called Ridge Gap Waveguide (RGW) has been investigated. The main objective of this work is to find a way to facilitate integration of active electronics with the gap waveguide structure and also to do measurements of the components made in gap waveguide technology working around $100 \mathrm{GHz}$. The transition design and simulation of $S$ parameters for the designed topology is presented.
\end{abstract}

Keywords-component; CPW; Microstrip; Perfect Magnetic Conductor (PMC); Artificial Magnetic Conductor (AMC); Gap Waveguide; transition; packaging.

\section{INTRODUCTION}

The gap waveguide emerged from the concept of artificial soft and hard surfaces in electromagnetics [1]. The theoretical principle of this technology was introduced in [2] and [3], while first experimental demonstrations are presented in [4] and [5] validating its characteristics in the microwave region with a potential for being more advantageous for millimeter applications.

This new guiding structure is realized in the gap between two metal parallel plates. One of them has a textured surface made by means of a periodical pattern which acts as an Artificial Magnetic Conductor (AMC). The AMC is the artificial realization of the Perfect Magnetic Conductor (PMC) and it establishes a high impedance condition within a certain frequency range. This means that the textured surface forbids the propagation of normal parallel plate modes in any direction as long as the distance to the opposing smooth plate is less than quarter wavelength. Still, local waves are allowed to propagate along metal ridge, strips or grooves present in the textured plate [3]. The frequency band where there is cut-off of parallel plate modes is called the stop band. A study of the achievable cutoff bandwidths by using different periodic structures can be found in [6].

The advantages of the gap waveguide technology as a new way for packaging passive microstrip circuits have been studied and demonstrated for packaging different components [7]-[9]. The characterization of the losses for this new kind of waveguide in terms of $\mathrm{Q}$ factor has been described in [10]. Real gap waveguide components (power dividers, couplers) have been lately implemented and demonstrated by measurements [11]. The gap waveguide technology results to be very promising for $\mathrm{THz}$ applications and integration of active components. This technology is very suitable at these frequencies since it is a low loss solution and there is no need of conducting joints facilitating its fabrication. The first millimeterwave demonstrators of RGW were manufactured by micromachining (MEMS technology) that is more accurate than milling at frequencies above $100 \mathrm{GHz}$ [12]. The main concern is that the measurements of these prototypes are difficult due to the incompatibility between the RGW and the available probe stations. Therefore, good transitions should be designed in order to take measurements in an accurate way.

The coplanar waveguide (CPW) technology is used as an alternative to the microstrip line for being a less dispersive and low cost solution. One positive aspect of the CPW is that both ground pads and signal pad are located on the same substrate side making it easier to integrate active and passive components into CPW than into microstrip [13]-[15]. In microstrip, via holes are needed if a component has to be connected to the ground plane.

One of the drawbacks regarding the utilization of CPW is the potential presence of parallel-plate modes if the ground side-planes are not connected with vias to the bottom ground plane (Conductor Backed $\mathrm{CPW}$ or $\mathrm{CBCPW}$ ). In the present study, a standard CPW without bottom ground plane has been considered in order to avoid the problem with the parallel-plate modes. However, there exists another disadvantage which is the mode coupling due to the odd and even modes generated by the ground side pads. This mode coupling can be very critical with the presence of discontinuities and asymmetries in the structure. Moreover, radiation leaking from the substrate material in the standard CPW has been observed. This could be a consequence from the mentioned mode coupling and it is a part of this work to overcome this problem via a gap waveguide solution.

The motivation of this work is to find good transitions that ensure the compatibility between CPW and the RGW. This will enable integration of MMIC with gap waveguide structure and will also allow measurements of gap waveguide components with existing probe station technology for applications above $100 \mathrm{GHz}$. On the other hand, the design of gap waveguide antennas is in the process of being explored, particularly slot arrays. Therefore, it will be useful to have suitable transitions to work as feeding network of these antennas. 
In this paper, a vialess transition from $\mathrm{CPW}$ to $\mathrm{RGW}$ via capacitive coupling is analyzed. The transition design and geometry for this topology is presented, as well as simulation results in terms of S parameters.

\section{BACK-TO-BACK CPW-TO-RGW TRANSITION DESIGN AND GEOMETRY}

The transition geometry is composed of two parts: the RGW located upside down, and the CPW which is placed under the ridge. The center frequency is considered to be 100 GHz.

The front view and the dimensions for the RGW interface are shown in Fig. 1. With these dimensions, the RGW is able to work in a bandwidth from 55 to $134 \mathrm{GHz}$.

TACONIC TRF-45 (permittivity $\varepsilon_{\mathrm{r}}=4.5$ ) is the dielectric material chosen for this transition design. The substrate thickness is $200 \mu \mathrm{m}$ and the copper thickness is $18 \mu \mathrm{m}$. The dissipation factor for this substrate is $\tan \delta=0.0035$. Input and output feeding CPW lines are added and designed in such a way that around $50 \Omega$ characteristic impedance is ensured.

The design of this transition consists of a CPW rectangular patch which is separated from the upper ridge by the air gap g. The CPW is placed so that its ground side-planes are extended constituting at the same time the metal plate used to form the gap of the gap waveguide together with the ridge (see Fig. 2).

In this first transition prototype, the length of the rectangular patch was initially taken as $\lambda \mathrm{g} / 2$, where $\lambda \mathrm{g}$ is the guided wavelength of the $\mathrm{CPW}$ line. The $\mathrm{CPW}$ patch couples the signal to the upper ridge without the need of any vias. An optimization of the patch parameters was made in order to improve the matching. Moreover, the ridge is divided into three parts. One central ridge section guides the coupled signal to the other side of the circuit, and it has a length of around $2 \lambda$. Other two sections are located at each side of the previous central ridge respectively. They are the responsible to receive/couple the signal from/to the CPW patches. Better performance in terms of return losses was observed when these two ridge sections follow the shape of the CPW patch (shrinking its width).

Two extra pins have been added before and after the ridge. Their function is to keep the input and output feeding lines packaged. This can avoid any possible unwanted radiation from the CPW circuit (see Fig. 3.). The resulting dimensions for this transition are represented in Fig. 4.

\section{SimULATIONS OF BACK-TO-BACK CPW-TO-RGW TRANSITION}

A numerical study of this transition in terms of $\mathrm{S}$ parameters has been performed by using CST Microwave Studio 2011[16].

Initially, the boundary condition which was considered below the substrate was open. Results were not satisfactory since the transition behavior was degraded due to the presence of radiation leaking away from the dielectric layer. Instead, the boundary condition that has been taken into account under the dielectric material is $\mathrm{PMC}$, in order to avoid possible radiation

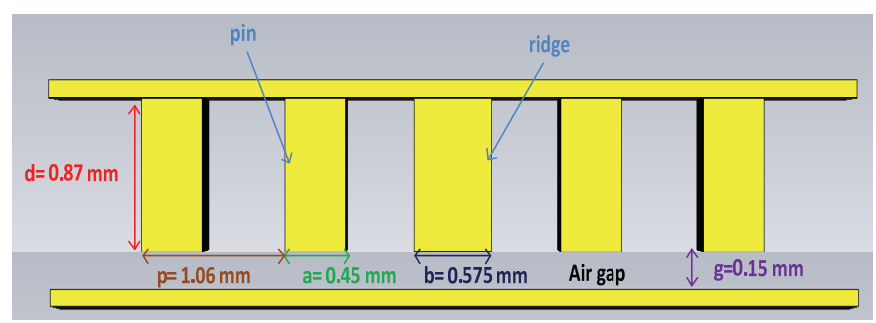

Figure 1. Front view of the ridge gap waveguide located upside down
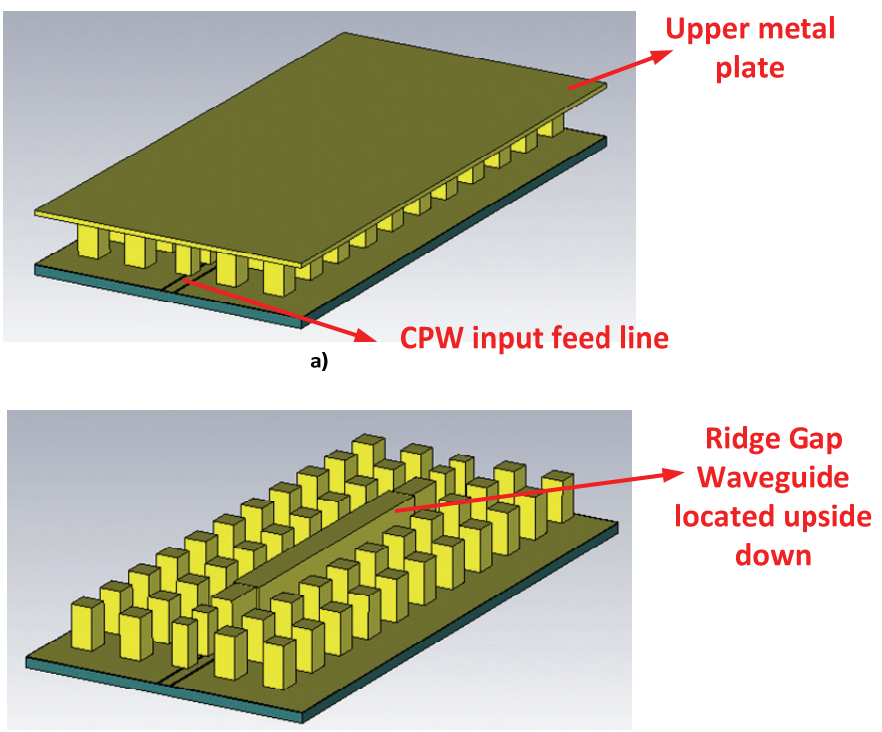

b)

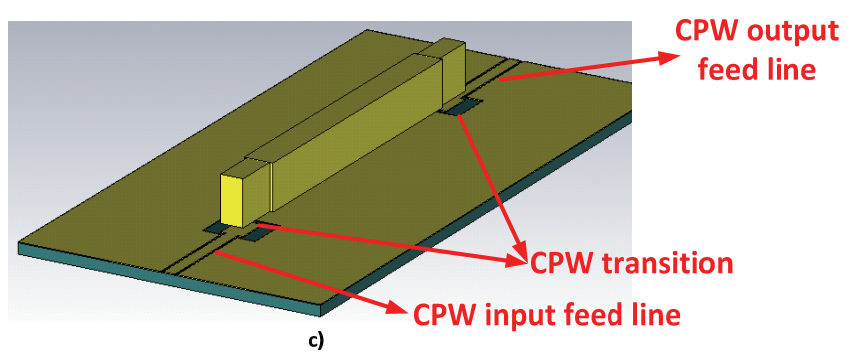

Figure 2. a) Complete topology of two back-to-back CPW-to-RGW transitions

b) Topology when upper metal plate is removed, except for its texture (the pins are kept). The RGW is located upside down c) Pins are removed as well in order to visualize the CPW transitions under the RGW

away from the substrate (see simulation results of $\mathrm{S}$ parameters in Fig. 5).

The simulation results show that return losses are better than $-15 \mathrm{~dB}$ over $10 \%$ bandwidth. The insertion loss is around $-1 \mathrm{~dB}$ from 100 to $108 \mathrm{GHz}$; ie, implying $0.5 \mathrm{~dB}$ loss for a single transition.

Fig. 6 shows the distribution of the vertical electric field component in the middle of the air gap and inside the dielectric. Some fields remain in the substrate and some fields are coupled to the side ridge sections and propagate through the main ridge.

In order to eliminate the radiation problem, the PMC boundary condition has been artificially realized by gap 


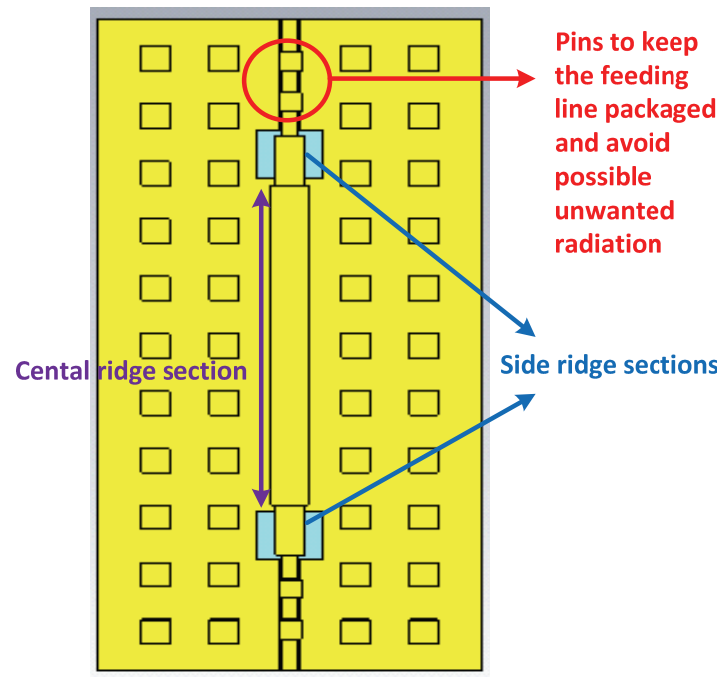

Figure 3. Top view of the circuit without the upper metal plate

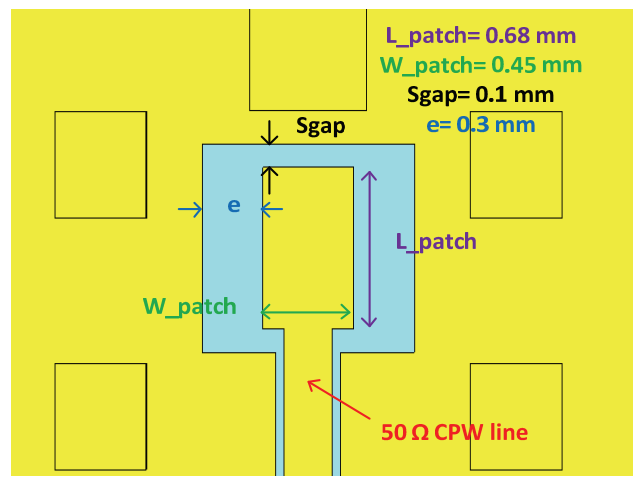

a)

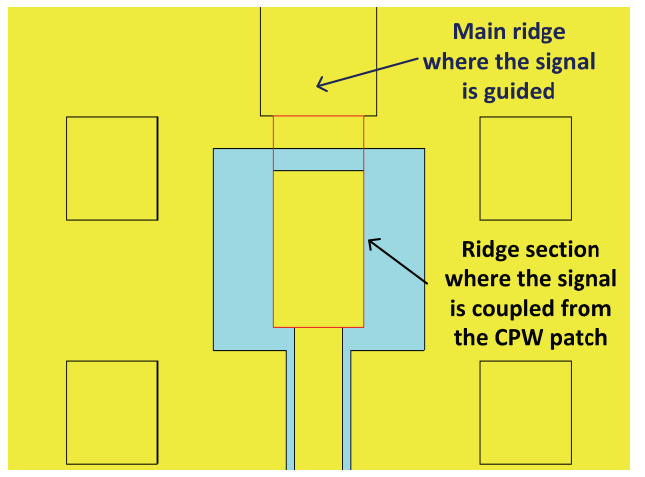

b)

Figure 4. a) Main parameters of the $\mathrm{CPW}$ rectangular patch

b) Ridge section in which the signal is coupled from the CPW patch

waveguide technology. This is explained and investigated in the next section where new simulations are computed and compared with the ideal PMC situation.

\section{REALIZATION OF PMC BOUNDARY FOR TRANSITION CPW-TO-RGW AND SIMULATIONS}

Our goal is to remove the radiation leaking away from the dielectric material which has been reported when there is open boundary underneath. We have previously demonstrated the

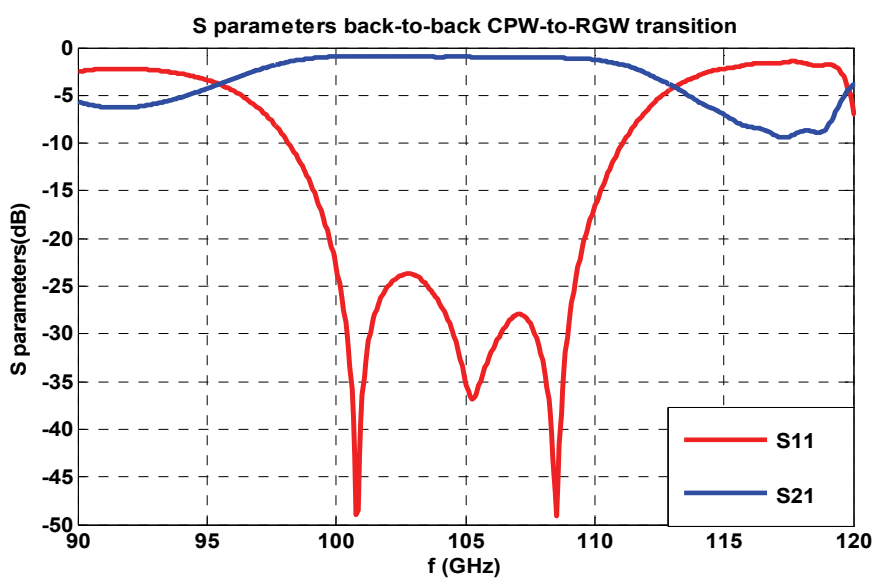

Figure 5. Computed S parameters of back-to-back arrangement of two CPWto-RGW transitions with PMC boundary condition underneath

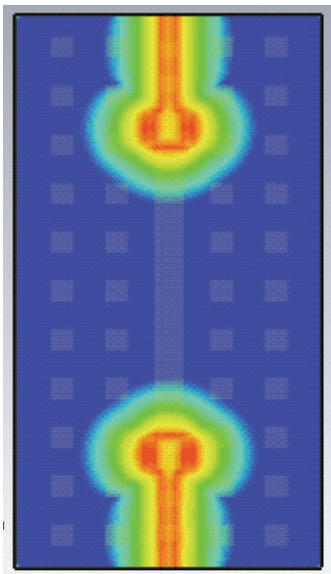

a)

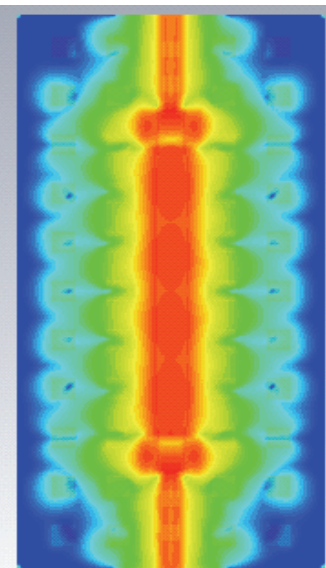

b)
Figure 6. a) Vertical field distribution inside the substrate

b) Vertical field distribution in the middle of the air gap

capability of the bed of nails to remove efficiently the radiation losses in microstrip circuits [7]-[9]. The same PMC packaging principle can be applied in this situation. There is a starting ideal situation which needs to be artificially realized via gap waveguide (check Fig.7).

The design of the pin dimensions for the bed of nails underneath the transition is based on the study of its working frequency bandwidth. The RGW upper interface and the bed of nails need to overlap their operating bandwidths to make the whole structure work properly. The dispersion diagram for both gap waveguide layers were computed with CST Eigenmode Solver (see Fig. 8). For the lower bed of nails, a stop band which covers a frequency band from 43.8 to 124 $\mathrm{GHz}$ is obtained if the pin dimensions are: pin width $\mathrm{a}=0.2$ $\mathrm{mm}$, period $\mathrm{p}=0.9 \mathrm{~mm}$ and pin height $\mathrm{d}=0.87 \mathrm{~mm}$. This stop band overlaps the bandwidth of the upper RGW, thus the whole structure should work in the band of interest. The final structure is illustrated in Fig. 9, where both gap waveguide layers are added.

The obtained S parameters for the complete topology (considering back-to-back transition again) and the vertical component of the electric field are represented in Fig. 10 and 


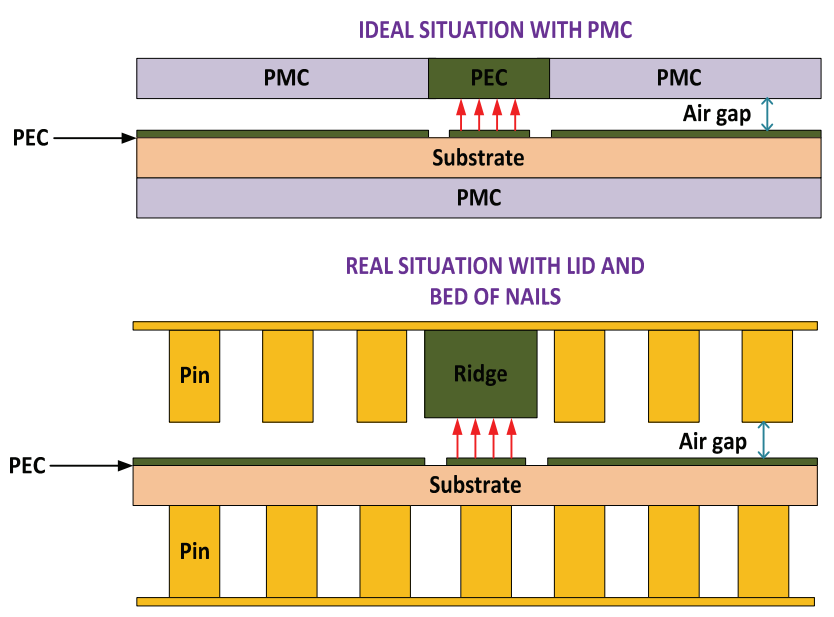

Figure 7. Realization of PMC boundary condition via gap waveguide

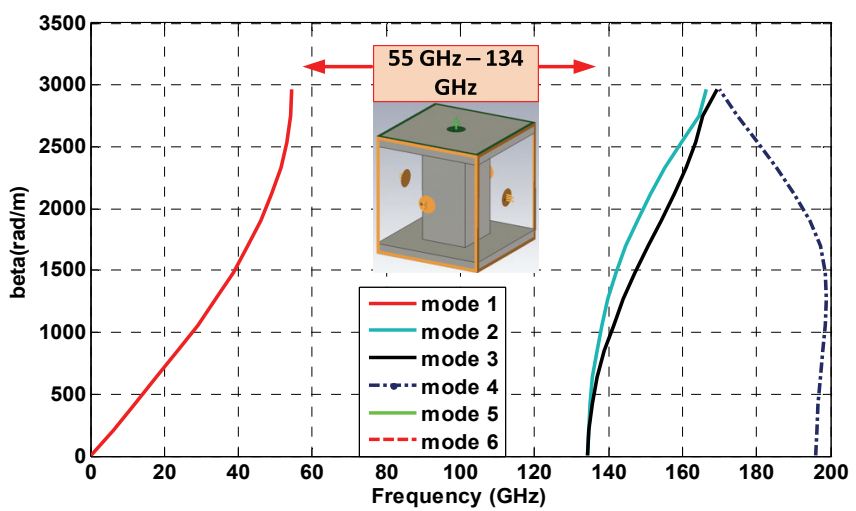

a)

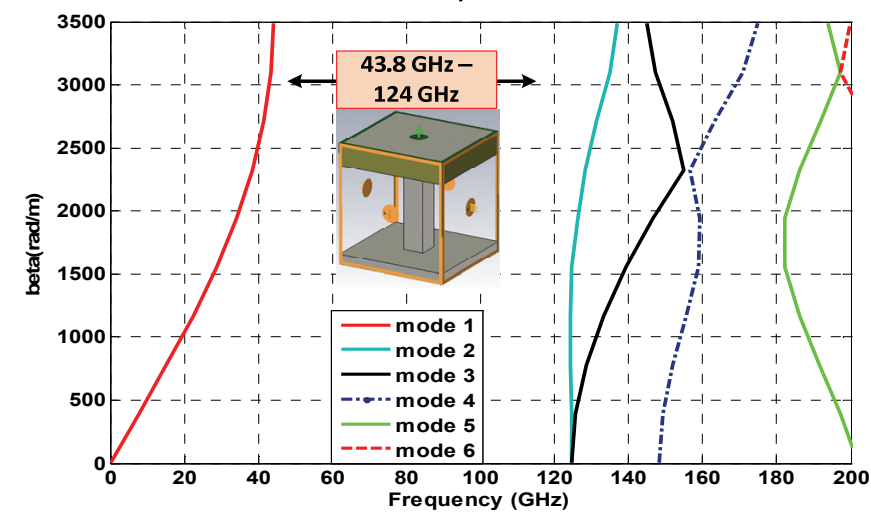

b)

Figure 8. a) Dispersion diagram for upper RGW layer

b) Dispersion diagram for lower RGW layer

Fig. 11 respectively. The return loss is better than $-15 \mathrm{~dB}$ over $9.5 \%$ bandwidth and the insertion loss is around $-1 \mathrm{~dB}$ from 99.3 $\mathrm{GHz}$ to $108.5 \mathrm{GHz}$. A relocation of the central pins (the ones placed before and after the ridge) was done in order to improve the matching. Also, a small tuning of the width of the patch was realized, modifying this parameter from 0.45 to $0.46 \mathrm{~mm}$.

One advantage of this device is the inherent suppression of the radiation leaking from the substrate by using gap waveguide technology. If this radiation is not removed the

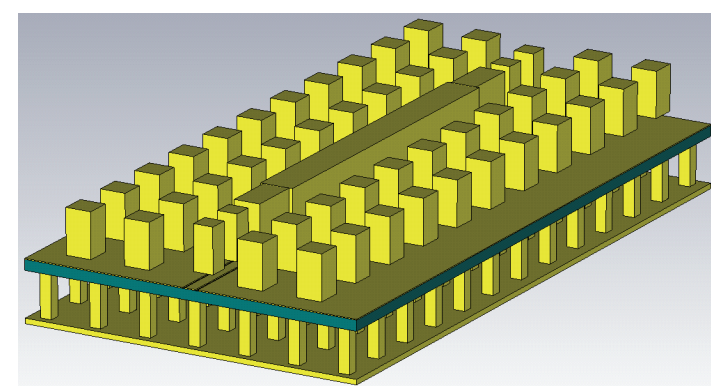

a)

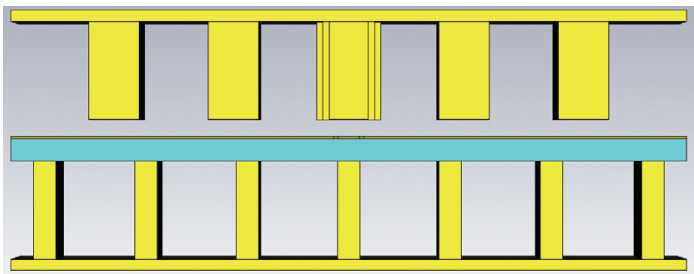

b)

Figure 9. a) Perspective view of the transition prototype with bed of nails below the dielectric (upper metal plate is removed)

b) Front view of the transition prototype with bed of nails below the dielectric.

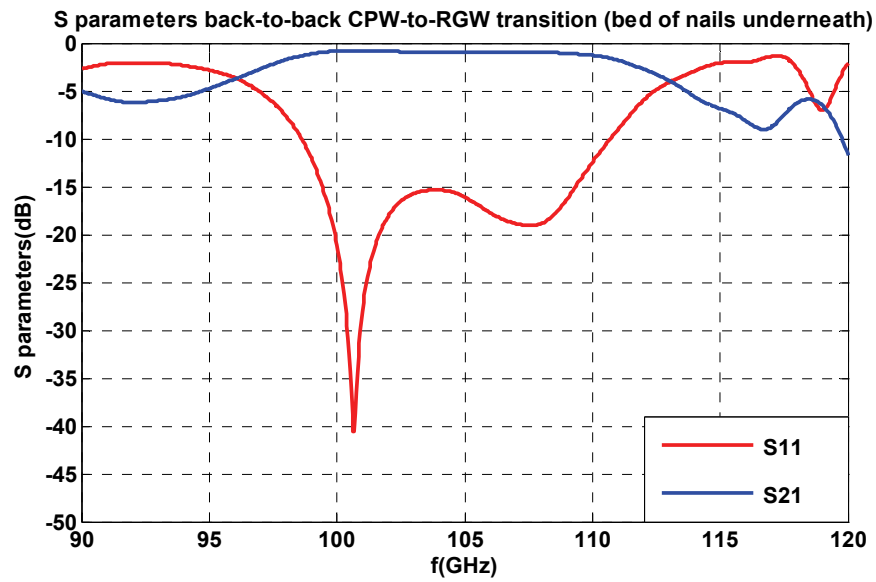

Figure 10. Computed $\mathrm{S}$ parameters of back-to-back configuration of two CPW-to-RGW transitions with bed of nails below dielectric material (realization of PMC boundary condition)

transition behavior presents resonances and degradation of the return and insertion loss. Another positive aspect is the mechanical support given by the bed of nails underneath.

\section{CONCLUSIONS}

A vialess CPW-to-RGW transition has been proposed and analyzed back-to-back. In order to suppress the leaking of radiation below the substrate, two transition cases are studied. Firstly, ideal PMC boundary condition is considered below the dielectric material. In the second case, an artificial realization of this PMC condition by using gap waveguide has been developed. Performances in terms of $\mathrm{S}$ parameters and field distributions have been presented.

We have found similar transition behavior in both cases. The resulting back-to-back return loss for the CPW-to-RGW transition with PMC condition is better than $-15 \mathrm{~dB}$ in around 

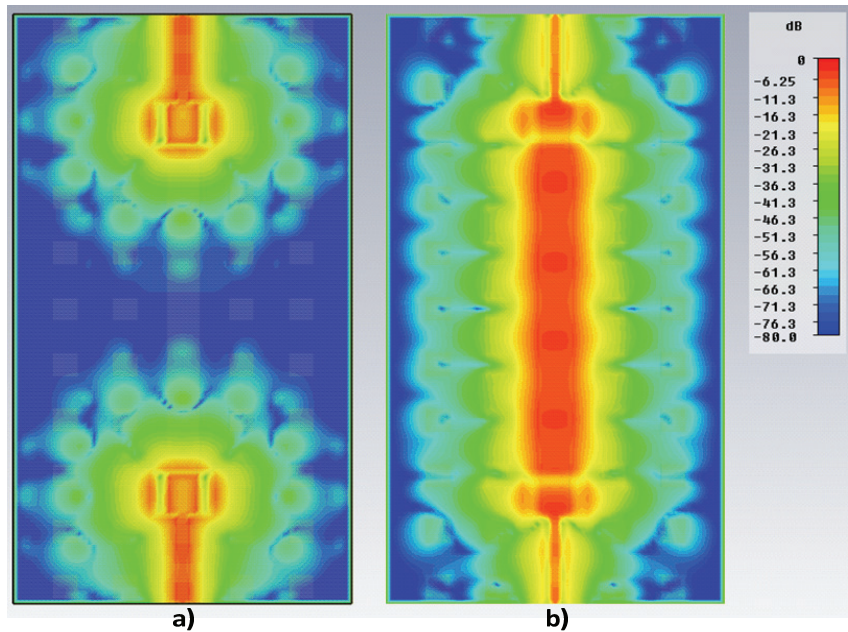

Figure 11. a) Vertical field distribution inside the substrate

b) Vertical field distribution in the middle of the air gap

$10 \%$ bandwidth. For the transition with PMC realization, the $\mathrm{S}_{11}$ parameter is less than $-15 \mathrm{~dB}$ in $9.5 \%$ bandwidth approximately. The value of insertion loss is less than $-1 \mathrm{~dB}$ for almost same frequency band in both situations. Since we have analyzed back-to-back transitions, the contribution to the insertion loss from a single transition would be smaller than $0.5 \mathrm{~dB}$. The back-to-back configuration also means that $\mathrm{S} 11$ of each transition may be as much as $6 \mathrm{~dB}$ lower than the interference peaks shown in the graphs, easily seen by using theory of small reflections, see Sec. 2.7 in [17] .

The mode coupling between the even and odd mode in the CPW (very critical with the presence of discontinuities and assymmetries), the radiation leaking away from the substrate, and the potential excitation of higher order modes might make the design of the CPW-to-RGW transition complex and time consumming. Therefore, the next step of this work is to design another type of transition, from microstrip-to-RGW, and compare both performances.

\section{ACKNOWLEDGMENT}

This work has been supported mainly by the Swedish Research Council VR, but also partly by Swedish Strategic Research Foundation (SSF) within the Chalmers Microwave Antenna Systems Research Center CHARMANT, and by the Swedish research council VINNOVA via a project within the VINN Excellence center Chase. The authors would also like to thank Prof. Vessen Vassilev from the Microtechnology and Nanoscience department at Chalmers University of Technology for his very useful advices and help to this project.

\section{REFERENCES}

[1] P.-S. Kildal, "Artificially soft and hard surfaces in electromagnetics", IEEE Transaction on Antennas and Propagation, vol. 28, no. 10, pp. 1537-1544, 1990.

[2] P.-S. Kildal, E. Alfonso, A. Valero-Nogueira, E. Rajo-Iglesias, "Local metamaterial-based waveguides in gaps between parallel metal plates" IEEE Antennas and Wireless Propagation Letters, vol. 8, pp.84-87, 2009.

[3] P.-S. Kildal, "Three metamaterial-based gap waveguides between parallel metal plates for $\mathrm{mm} / \mathrm{submm}$ waves", $3 r$ European Conference on Antennas and Propagation EUCAP 2009, Berlin, Germany, 23-27 March 2009.

[4] A. Valero-Nogueira, E. Alfonso, J. I. Herranz, P.-S. Kildal, "Experimental demonstration of local quasi-TEM gap modes in singlehard-wall waveguides", IEEE Microwave and Wireless Components Letters, Vol. 19, No. 9, pp. 536-538, Sept. 2009.

[5] P.-S. Kildal, A. Uz Zaman, E. Rajo-Iglesias, E. Alfonso, A. ValeroNogueira, "Design and experimental verification of ridge gap waveguides in bed of nails for parallel plate mode suppression", IET Microwaves, Antennas \& Propagation, Vol. 5, No 3, pp. 262-270, March 2011..

[6] E. Rajo-Iglesias, P.-S. Kildal, "Numerical studies of bandwidth of parallel plate cut-off realized by bed of nails, corrugations and mushroom-type EBG for use in gap waveguides", IET Microwaves, Antennas \& Propagation, Vol. 5, No 3, pp. 282-289, March 2011.

[7] E. Rajo-Iglesias, A. Uz Zaman, P.-S. Kildal, "Parallel plate cavity mode suppression in microstrip circuit packages using a lid of nails", IEEE Microwave and wireless Components Letters, Vol. 20, No. 1,pp. 31-33, Dec. 2009.

[8] A.Uz Zaman, J.Yang, P.-S. Kildal, "Using Lid of Pins for Packaging of Microstrip Board for Descrambling the Ports of Eleven Antenna for Radio Telescope Applications", 2010 IEEE Antennas and Propagation Society International Symposium, pp. 1-4, 2010.

[9] A. Algaba Brazález, A. Uz Zaman, P.-S. Kildal, "Improved microstrip filters using PMC packaging by lid of nails", accepted for publication in IEEE Transactions on Components, Packaging and Manufacturing Technology, October 2011.

[10] E. Pucci, A. Uz Zaman, E. Rajo-Iglesias, P.-S. Kildal, A. Kishk, "Losses in ridge gap waveguide compared with rectangular waveguide and microstrip transmission lines", 4th European Conference on Antennas and Propagation EUCAP 2010, Barcelona, Spain, 12-16 April 2010.

[11] E. Alfonso, M. Baquero, P.-S. Kildal, A. Valero-Nogueira, E. RajoIglesias, J.I. Herranz, "Design of Microwave circuits in ridge-gap waveguide technology", 2010 IEEE MTT-S Microwave symp. digest, pp. 1544-1547, 2010.

[12] S. Rahiminejad, A.Uz Zaman, E. Pucci, H. Raza, V. Vassilev, S. Haasl, P. Lundgren, P.-S. Kildal, P. Enoksson, "Design of Micromachined Ridge Gap Waveguides for Millimeter-Wave Applications", Proc. Eurosensors XXV, September 4-7, 2011, Athens, Greece.

[13] Rainee N. Simons, Coplanar Waveguide Circuits, Components, and Systems, Wiley, 2002.

[14] Jackson, R.W., "Considerations in the use of coplanar waveguide for millimeter-wave integrated circuits", IEEE Transaction on Microwave Theory and Techniques, vol. 34, no. 12, pp. 1450-1456, 1989.

[15] D. Pozar, Microwave Engineering, $3^{\text {rd }}$ edition, Wiley, 2005.

[16] CST Microwave Studio 2011, www.cst.com.

[17] P.-S. Kildal, Foundations of Antennas - A Unified Approach, Studentlitteratur, Lund, Sweden, 2000. 